\title{
Abundance and Diversity Index of Weeds in Oil Palm and Vegetable Intercropping in Rainforest Zone of Nigeria
}

\author{
Ayodele Samuel Oluwatobi ${ }^{1 *}$ and Kehinde Stephen Olorunmaiye ${ }^{2}$ \\ ${ }^{1}$ Department of Natural and Environmental Sciences, Faculty of Science, Crown-Hill University, \\ Ilorin, Nigeria; ${ }^{2}$ Department of Plant Biology, Faculty of Life Sciences, \\ University of Ilorin, Ilorin, Nigeria \\ *Corresponding author: ayodeleoluwatobi@gmail.com
}

\begin{abstract}
The problem of severe weed infestation often arises during the early phases of establishment of oil palm field due to the spacing requirement and growth habit of young oil palm plantation until later years when the canopy closes. This study was conducted at Ala, Akure-North Local Government Area, Ondo State, Nigeria, to investigate the composition of weed species and their distribution in fruit vegetable-juvenile oil palm intercrop. The fallow alleys within the immature oil palm were intercropped with 2 accessions of tomato (NGB 01665 and NG/AA/SEP/09/053) and eggplant (NGB 01737). The sampling of the weed species was carried out with a quadrat $\left(0.25 \mathrm{~m}^{2}\right)$. Weed species parameters and the Diversity Index (D) were quantitatively analyzed. The results revealed that members of Asteraceae and Poaceae gave the highest weed species at 3 and 6 weeks after intercropping (WAI) (17.857\% and 19.04\%) respectively. A total of 23 and 16 were found at 3 and $6 \mathrm{WAI}$, while the least diversity index of 0.734 was recorded in the immature oil palm/tomato (NGB 01665) plot at 6 WAI. Farmers should be persuaded to simultaneously intercrop fruit vegetables within the alley of juvenile oil palm, particularly at the earlier years prior to closure of the oil palm canopy.
\end{abstract}

Keywords: distribution; diversity; intercrop; oil palm; weed

Cite this as: Oluwatobi, A. S., \& Olorunmaiye, K. S. (2021). Abundance and Diversity Index of Weeds in Oil Palm and Vegetable Intercropping in Rainforest Zone of Nigeria. Caraka Tani: Journal of Sustainable Agriculture, 36(2), 227-237. doi: http://dx.doi.org/10.20961/carakatani.v36i2.48098

\section{INTRODUCTION}

The standard spacing requirement of $6-9 \mathrm{~m}$ deployed during the field cultivation of oil palm gives room for a waste of solar radiation and has resulted in severe weed invasion from field transplanting to the stage of canopy closure when the palms become fruitful. During the long juvenile phase, most farmers expend a lot of their capital on labor cost to control weeds in the plantation yearly without any significant monetary returns (Oluwatobi et al., 2020). Weeds compete with main crops for nutrients, water, sunlight and space when these resources are limited. They reduce the utilization of fertilizer, interfere with and cut down the production of main crops (Suryaningsih and Darmadi, 2011; Susanti and Safrina, 2018). Ali et al. (2014) documented that weeds constitute the most serious and extensive biological limitation on crop production and cause significant damage until the crops are harvested.

Weeds also cause inhibitory activity on the germination parameters of desired crops (Susanti et al., 2021). The fallow and empty alley within the newly established oil palm plantation, consequent of the pattern of spacing, has culminated in underutilization of

\footnotetext{
* Received for publication January 26, 2021

Accepted after corrections March 24, 2021
} 
the environmental resources such as space, $\mathrm{CO}_{2}$, sunlight, water, etc. (Rezig et al., 2012). Therefore, the introduction of annual crops such as vegetables, groundnut and soybeans into the fallow alley in the young oil palm plantation would be advisable, as it would furnish the farmers with the opportunity to improve the land productivity and facilitate proficient deployment of the environmental resources (Amanullah et al., 2006; Abera and Feyisa, 2008; Aynehband et al., 2010; Rezig et al., 2012).

Tree canopy encourages surface litter accumulation and promotes soil fertility (Livesley et al., 2016; Rahayu et al., 2020). Many grass species thrive well and exhibit good traits under sunny environmental condition, contrary to what is obtainable under shaded environment (Rahayu et al., 2020). Shade lessens solar radiation and influences the microclimate around the environment (Rahayu et al., 2020). Growth of weed species is hindered as a result of reduction in photosynthetic activities, $\mathrm{CO}_{2}$ generation and other aesthetic features when they are subjected to shade condition (Okeyo et al., 2014; Rahayu et al., 2020). Reduced light situation contributes to fewer green coverage because tree leaves sort the photosynthetically vital red and blue light wavelengths before they reach weed leaf surfaces. Various plant species possess varying shade tolerance and rate of responses to the intensity of sunlight received (Peterson et al., 2014; Audina et al., 2016). The reaction of plants to the intensity of solar radiation differs according to the species; that is shade or full sun plants (Rahayu et al., 2020). Plants with full sun conditions tend to have higher root dry weight than those subjected to shade conditions. At low light intensity, shade tolerant plants can accumulate photosynthetic products than those grown in full sunlight (Pantilu et al., 2012).

The kind of weeds and their density are one of the major factors that influence the competition for resources and performance of desired crops (Susanti et al., 2021). Weed management approaches like using cover crops to smother weeds, seedbed treatment or tillage operations may be used for additional efficient weed management in integrated and organic farming systems (Devkota and Jha, 2010; Kanatas et al., 2020). Evaluation of the impacts of the various intercropping arrangements, coupled with the component crop mixtures on the distribution of weed is crucial in identifying a better intercropping models and crop mixtures that would contribute to the alleviation of weeds infestation tremendously, coming up with superior weed management system, increased yield and ultimately and support sustainable agriculture. Irrespective of the cropping designs employed, weed concern remains a critical cause of yield loss during cultivation. Thus, comprehensive knowledge and understanding of their distribution and mechanism of survival would facilitate further studies to diminish distressing impacts of weeds to agricultural farms (Oluwatobi et al., 2020).

The number of weed species within a community could be used to measure the distribution of weed species present with regards to the species richness. Weed species distribution maybe between or within communities; as two communities that share the same number of species may vary in terms of species evenness and thus it is valuable to have the knowledge of the proportion or relative species abundance found in the community (Karaye et al., 2007). Therefore, this study was aimed at determining the composition and diversity index of weed species within the intercropped alleys and control plot.

\section{MATERIALS AND METHOD}

\section{Study area}

\section{Description of study area}

The study was conducted during the rainy season of 2017 within an established 2 yearold oil palm plantation situated at Ala, AkureNorth Local Government Area, Ondo State, Nigeria. The plantation can be found at a coordinate between longitude $5^{\circ} 21^{\prime} 15.19177$ '5'21'15.47179' "E and latitude 7'5'34.8857'$7^{\circ} 5^{\prime} 35.59837^{\prime \prime} \mathrm{N}$, in the tropical rain forest region of Nigeria. Variation in annual rainfall is between 1,150 to $2,550 \mathrm{~mm}$, while the temperature is described to be moderately high year-round, with a range of $22-34{ }^{\circ} \mathrm{C}$ (Ogunrayi et al., 2016). Seeds of tomato accessions (NGB 01665 and NG/AA/SEP/09/053) and eggplant (NGB 01737) obtained from National Centre for Genetic Resources and Biotechnology, Ibadan, Oyo State, Nigeria, were transplanted separately into the alleys of the juvenile oil palm plantation after being raised in the nursery for 4 weeks.

The soil $\mathrm{pH}$ is slightly acidic. Organic matter content and cation exchange capacity 
ranges are $2.317-4.565 \mathrm{~g} \mathrm{~kg}^{-1}$ and $2.439-4.298 \%$, respectively.

\section{Collection of weed species}

The survey of weed species was carried out within each plot by following the methods described by (Olorunmaiye et al., 2011). An 'M' guide-pattern for collection of weeds was precisely identified and mapped out in each of the plots and $0.25 \mathrm{~m}^{2}$ quadrat was used for sampling. Collected data were subjected to ecological analysis (Oluwatobi and Olorunmaiye, 2014; Dingaan and Du Preez,
2017). Weeds were identified using a standard Handbook of West African Weeds (Akobundu and Agyakwa, 1998).

Weed species parameters and diversity indices

The parameters were calculated according to methods of Olorunmaiye et al. (2013) and Oluwatobi and Olorunmaiye (2014). Weed frequency was calculated as number of quadrats where weed species was found. The density of weeds was calculated as number of species per area of quadrat. Determinations of other parameters were as follows:

$$
\begin{array}{ll}
\text { Relative frequency } & =\frac{\text { frequency of species }}{\text { total frequency of all species }} \times 100 \\
\text { Relative density } & =\frac{\text { density of species }}{\text { total density of all species }} \times 100 \\
\text { Relative importance value (RIV) } & =\text { relative frequency }+ \text { relative density } \\
\text { Abundance } & =\frac{\text { relative frequency }+ \text { relative density }}{2} \\
\text { Dominance } & =\frac{\text { absolute density of species }}{\text { number of quadrats where species are found }} \times 100
\end{array}
$$

Simpson's Diversity Index (D): This index indicates the chance that two individual weed species randomly selected from a sample will fit into the same species. This index was computed as follows:

$$
\text { Simpson's diversity index }(D)=\frac{\sum \mathrm{n}(\mathrm{n}-1)}{\mathrm{N}(\mathrm{N}-1)}
$$

Where:

$\mathrm{n}=$ total number of a particular weed species;

$\mathrm{N}=$ total number of all weed species.

Simpson's index of diversity $(1-D)$ : This was computed by subtracting Simpson's diversity index (D) from 1.

Simpson's reciprocal index (1/D): This index started with 1 as the lowest probable figure. It was achieved by finding the inverse of Simpson's index of diversity (D) (Oluwatobi and Olorunmaiye, 2014).

\section{Data analysis}

The data gathered were computed using Microsoft Excel Package (2010) and the results were presented in tables.

\section{RESULTS AND DISCUSSION}

\section{Composition and distribution of weed species}

A total of 28 different weed species were recorded across the 4 plots surveyed at 3 and 6 WAI and belong to 19 families. Members of the Poaceae recorded the highest number (21.43\%), followed by members of Asteraceae and Euphorbiaceae (21.43\% and $7.14 \%)$ respectively. Other families each of which has one member present (3.57\%) are Campanulaceae, Cleomaceae, Commenlinaceae, Convolvulaceae, Cyperaceae, Lamiaceae, LeguminosaePapilionoideae, Loganaceae, Malvaceae, Nyctaginaceae, Phyllanthaceae, Piperaceae, Portulaceae, Rubiacea, Solanaceae and Urticaceae. Broadleaf weeds represent $75 \%$ of the weed species encountered during the survey, while grasses and sedge represent $21.43 \%$ and $3.57 \%$ respectively (Table 1 - Table 8 ).

The decline observed in number of weed species at 3 through to 6 WAI may be ascribed to the combined shade effect of the 2-year-old oil palm and the fruit vegetables. This observation agrees with the submissions of Shadiul et al. (2011) and Ademabayoje et al. 
(2020). The impact of soil tillage carried out in the fallow alley before intercropping may be responsible for the reduction in the number of weeds. This thought is in tandem with earlier report that tillage operation enhances weed suppression capability (Lawson et al., 2006; Kanatas et al., 2020). This concurs with the submission of Baker et al. (2018) that weed seeds amass in the top soil in sustainable agriculture-in which minute or reduced tillage was practiced. This corroborates an earlier study by Weber et al. (2017), who attributed prompt weed growth to the accessibility of favourable germination conditions at the top soil.
Significantly elevated broadleaf weeds were reported in many of the intercropped plots in comparison to grasses and this may be ascribed to the abundance of weed propagules in seedbank of shaded soil in (Sherifi and Berisha, 2019; Ademabayoje et al., 2020). Canopy cover presented by the immature oil palm could translate to diminished light availability, thus promoting the growth of broadleaf weeds. Olorunmaiye et al. (2011) reported high concentration of broadleaf weeds underneath the canopies of adult citrus trees. Alridiwirsah et al. (2020) also reported similarly that there was abundance of broadleaf weeds in guava plants (monocrop) of $26.11 \%$.

Table 1. Weed species distribution parameters in tomato (NGB 01665)-oil palm intercropped plot at 3 WAI

\begin{tabular}{|c|c|c|c|c|c|c|c|c|c|}
\hline No. & Weed species & $\stackrel{\bar{\pi}}{0}$ & 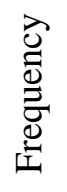 & 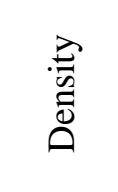 & 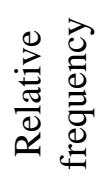 & 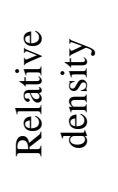 & 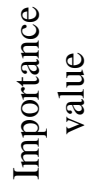 & 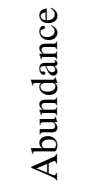 & 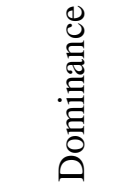 \\
\hline 1. & Ageratum conizoides Linn. & 35 & 5 & 28.0 & 9.4 & 6.7 & 16.1 & 8.1 & 560.0 \\
\hline 2. & Borreria ocymoides (Burm. f.) DC. & 193 & 5 & 154.4 & 9.4 & 36.9 & 46.3 & 23.2 & $3,088.0$ \\
\hline 3. & $\begin{array}{l}\text { Brachiaria deflexa (Schumach.) } \\
\text { C.E. Hubbard ex Robyns }\end{array}$ & 26 & 4 & 26.0 & 7.5 & 6.2 & 13.8 & 6.9 & 650.0 \\
\hline 4. & Cleome rutidosperma DC. & 1 & 1 & 4.0 & 7.5 & 1.0 & 8.5 & 4.3 & 400.0 \\
\hline 5. & Commelina benghalensis L. & 28 & 5 & 22.5 & 9.4 & 5.4 & 14.8 & 7.4 & 450.0 \\
\hline 6. & Digitaria horizontalis Willd. & 53 & 5 & 43.2 & 9.4 & 10.3 & 19.8 & 9.9 & 864.0 \\
\hline 7. & Euphorbia heterophylla Linn. & 4 & 3 & 5.3 & 5.7 & 1.3 & 6.9 & 3.5 & 177.7 \\
\hline 8. & Euphorbia hirta Linn. & 10 & 4 & 10.0 & 7.5 & 2.4 & 9.9 & 5.0 & 250.0 \\
\hline 9. & Evolvulus alsinoides (Linn.) Linn. & 29 & 5 & 23.5 & 9.4 & 5.6 & 15.1 & 7.5 & 470.0 \\
\hline 10. & $\begin{array}{l}\text { Mariscus alternifolius Vahl. (=M. } \\
\text { umbellatus Vahl.) }\end{array}$ & 76 & 5 & 60.8 & 9.4 & 14.5 & 24.0 & 12.0 & $1,216.0$ \\
\hline 11. & Paspalum conjugatum Berg. & 4 & 3 & 5.3 & 5.7 & 1.3 & 6.9 & 3.5 & 177.7 \\
\hline 12. & $\begin{array}{l}\text { Phyllanthus amarus Schum. \& } \\
\text { Thonn. }\end{array}$ & 39 & 5 & 31.2 & 9.4 & 7.5 & 16.9 & 8.4 & 624.0 \\
\hline 13. & Sida acuta Burm. F. & 3 & 3 & 4.0 & 5.7 & 1.0 & 6.6 & 3.3 & 133.3 \\
\hline
\end{tabular}

Table 2. Weed species distribution parameters in tomato (NGB 01665)-oil palm intercropped plot at $6 \mathrm{WAI}$

\begin{tabular}{|c|c|c|c|c|c|c|c|c|c|}
\hline No. & Weed species & $\begin{array}{l}\bar{\pi} \\
\stackrel{0}{0}\end{array}$ & 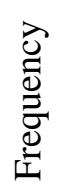 & $\begin{array}{l}\stackrel{\vec{D}}{0} \\
\overline{0} \\
0\end{array}$ & 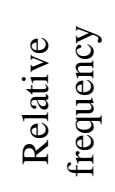 & 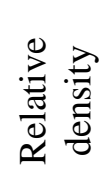 & 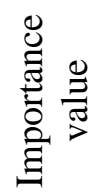 & 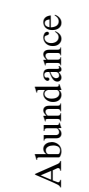 & 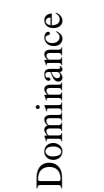 \\
\hline 1. & Ageratum conizoides Linn. & 39 & 5 & 31.2 & 10.9 & 7.6 & 18.5 & 9.2 & 624.0 \\
\hline 2. & Bidens pilosa Linn. & 31 & 4 & 31.0 & 8.7 & 7.6 & 16.3 & 8.1 & 775.0 \\
\hline 3. & Boerhavia diffusa $\mathrm{L}$. & 25 & 5 & 20.0 & 10.9 & 4.9 & 15.7 & 7.9 & 400.0 \\
\hline 4. & Borreria ocymoides (Burm. f.) DC. & 49 & 5 & 39.2 & 10.9 & 9.6 & 20.4 & 10.2 & 784.0 \\
\hline 5. & Commelina benghalensis L. & 2 & 2 & 4.0 & 4.3 & 1.0 & 5.3 & 2.7 & 200.0 \\
\hline
\end{tabular}


Table 2. Continued

\begin{tabular}{|c|c|c|c|c|c|c|c|c|c|}
\hline 6. & Croton lobatus L. & 5 & 3 & 6.7 & 6.5 & 1.6 & 8.1 & 4.1 & 222.2 \\
\hline 7. & Hyptis lanceolata Poir. & 3 & & 6.0 & 4.3 & 1.5 & 5.8 & 2.9 & 300.0 \\
\hline 8 & $\begin{array}{l}\text { Mariscus alternifolius Vahl. (=M. } \\
\text { umbellatus Vahl.) }\end{array}$ & 55 & 5 & 44.0 & 10.9 & 10.7 & 21.6 & 10.8 & 880.0 \\
\hline & $\begin{array}{l}\text { Peperomia pellucida (L.) H. B. } \\
\text { \& K. }\end{array}$ & 231 & & 8 & 10 & 45.1 & 55.9 & 28.0 & $3,696.0$ \\
\hline & Setaria barbata (Lam.) Kunth & 39 & & 31.2 & 10.9 & 7.6 & 18.5 & 9.2 & 624.0 \\
\hline & ta Burm. f. & 2 & & 4.0 & 4.3 & 1.0 & 5.3 & 2.7 & 200.0 \\
\hline & Solanum nigrum L. & 6 & & 8.0 & 6.5 & 2.0 & 8.5 & 4.2 & \\
\hline
\end{tabular}

Table 3. Weed species distribution parameters in tomato (NG/AA/SEP/09/053)-oil palm intercropped plot at 3 WAI

\begin{tabular}{|c|c|c|c|c|c|c|c|c|c|}
\hline No. & Weed species & 吾 & 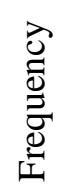 & 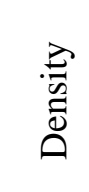 & 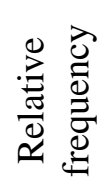 & 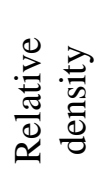 & 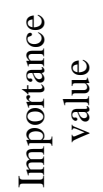 & 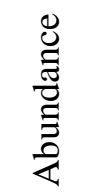 & 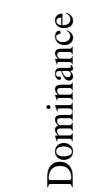 \\
\hline 1. & Ageratum conizoides Linn. & 62 & 5 & 49.6 & 9.6 & 16.5 & 26.1 & 13.0 & 992.0 \\
\hline 2. & $\begin{array}{l}\text { Axonopus compressus (Sw.) P. } \\
\text { Beauv. }\end{array}$ & 7 & 4 & 7.0 & 7.7 & 2.3 & 10.2 & 5.0 & 175.0 \\
\hline 3. & Bidens pilosa Linn. & 4 & 3 & 5.3 & 5.8 & 1.8 & 7.5 & 3.8 & 177.7 \\
\hline 4. & Borreria ocymoides (Burm. f.) DC. & 62 & 5 & 49.6 & 9.6 & 16.5 & 26.1 & 13.0 & 992.0 \\
\hline 5. & $\begin{array}{l}\text { Brachiaria deflexa (Schumach.) } \\
\text { C.E. Hubbard ex Robyns }\end{array}$ & 42 & 5 & 33.6 & 9.6 & 11.2 & 20.8 & 10.4 & 672.0 \\
\hline 6. & Digitaria horizontalis Willd. & 38 & 5 & 30.4 & 9.6 & 10.1 & 19.7 & 9.9 & 608.0 \\
\hline 7. & Euphorbia hirta Linn. & 1 & 1 & 4.0 & 1.9 & 2.3 & 3.3 & 1.6 & 400.0 \\
\hline 8. & Evolvulus alsinoides (Linn.) Linn. & 6 & 3 & 8.0 & 5.8 & 2.7 & 8.4 & 4.2 & 266.7 \\
\hline 9. & Hyptis lanceolata Poir. & 2 & 1 & 8.0 & 1.9 & 2.7 & 4.6 & 2.3 & 800.0 \\
\hline 10. & $\begin{array}{l}\text { Mariscus alternifolius Vahl. (=M. } \\
\text { umbellatus Vahl.) }\end{array}$ & 3 & 2 & 6.0 & 3.8 & 2.0 & 5.8 & 2.9 & 300.0 \\
\hline 11. & Paspalum conjugatum Berg. & 4 & 2 & 8.0 & 3.8 & 2.7 & 6.5 & 3.3 & 400.0 \\
\hline 12. & Pouzolzia guineensis Benth. & 2 & 2 & 4.0 & 3.8 & 1.3 & 5.2 & 2.6 & 200.0 \\
\hline 13. & Synedrella nodiflora Gaertn. & 50 & 5 & 40.0 & 9.6 & 13.3 & 22.9 & 11.4 & 800.0 \\
\hline 14. & Talinum triangulare (Jacq.) Willd. & 14 & 3 & 18.7 & 5.8 & 6.2 & 12.0 & 6.0 & 622.3 \\
\hline 15. & Tephrosia pedicellata Bak. & 4 & 2 & 8.0 & 3.8 & 2.7 & 6.5 & 3.3 & 400.0 \\
\hline & Tridax procumbens Linn. & 21 & 4 & 21.0 & 7.7 & 7.0 & 14.7 & 7.3 & 525.0 \\
\hline
\end{tabular}

Table 4. Weed species distribution parameters in tomato (NG/AA/SEP/09/053)-oil palm intercropped plot at 6 WAI

\begin{tabular}{|c|c|c|c|c|c|c|c|c|c|}
\hline No. & Weed species & $\stackrel{\pi}{0}$ & 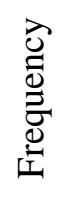 & 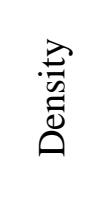 & 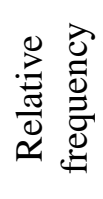 & 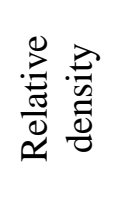 & 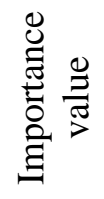 & 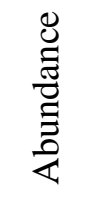 & 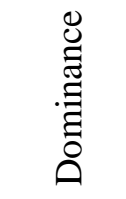 \\
\hline 1. & Ageratum conizoides Linn. & 67 & 5 & 53.6 & 12.2 & 20.6 & 32.8 & 16.4 & $1,072.0$ \\
\hline 2. & Bidens pilosa Linn. & 46 & 5 & 36.8 & 12.2 & 14.1 & 26.3 & 13.2 & 736.0 \\
\hline 3. & Borreria ocymoides (Burm. f.) DC. & 8 & 3 & 10.7 & 7.3 & 4.1 & 11.4 & 5.7 & 355.6 \\
\hline 4. & Commelina benghalensis L. & 45 & 5 & 36.0 & 12.2 & 13.8 & 26.0 & 13.0 & 720.0 \\
\hline 5. & Euphorbia hirta Linn. & 5 & 3 & 6.7 & 7.3 & 2.6 & 9.9 & 4.9 & 233.3 \\
\hline 6. & $\begin{array}{l}\text { Mariscus alternifolius Vahl. (=M. } \\
\text { umbellatus Vahl.) }\end{array}$ & 4 & 2 & 8.0 & 4.9 & 3.0 & 7.9 & 4.0 & 400.0 \\
\hline
\end{tabular}


Table 4. Continued

\begin{tabular}{|c|c|c|c|c|c|c|c|c|c|}
\hline & $\begin{array}{l}\text { Peperomia pellucida (L.) H. B. } \\
\text { \& K. }\end{array}$ & 41 & 5 & 32.8 & 12.2 & 12.6 & 24.8 & 12.4 & 656.0 \\
\hline 8. & $\begin{array}{l}\text { Phyllanthus amarus Schum. \& } \\
\text { Thonn. }\end{array}$ & 4 & 3 & 5.3 & 7.3 & 2.0 & 9.4 & 4.7 & 177.7 \\
\hline & Setaria barbata (Lam.) Kunth & 73 & & 58.4 & 12.2 & 22.4 & 34.0 & 17.3 & $1,168.0$ \\
\hline & Solanum nigrum $\mathrm{L}$. & 3 & & 4.0 & 7.3 & 1.5 & 8.9 & 4.4 & 133.3 \\
\hline & Talinum triangulare (Jacq.) Willd. & 4 & & 8.0 & 4.9 & 3.0 & 7.9 & 4.0 & 400.0 \\
\hline
\end{tabular}

Table 5. Weed species distribution parameters in eggplant (NGB 01737)-oil palm intercropped plot at 3 WAI

\begin{tabular}{|c|c|c|c|c|c|c|c|c|c|}
\hline No. & Weed species & $\stackrel{\pi}{0}$ & 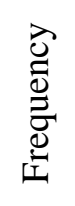 & $\begin{array}{l}\stackrel{2}{0} \\
\text { Dे } \\
\stackrel{0}{0}\end{array}$ & 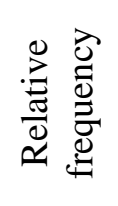 & 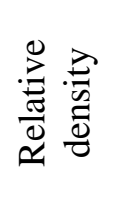 & 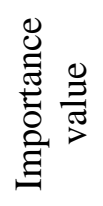 & 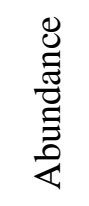 & 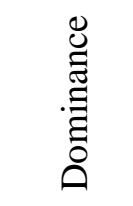 \\
\hline 1. & Ageratum conizoides Linn. & 24 & 4 & 24.0 & 6.5 & 5.8 & 12.3 & 6.1 & 600.0 \\
\hline 2. & Bidens pilosa Linn. & 3 & 2 & 6.0 & 3.2 & 1.5 & 4.7 & 2.3 & 300.0 \\
\hline 3. & Boerhavia diffusa $\mathrm{L}$. & 4 & 3 & 5.3 & 4.8 & 1.3 & 6.1 & 3.1 & 177.7 \\
\hline 4. & Borreria ocymoides (Burm. f.) DC. & 14 & 3 & 18.7 & 4.8 & 4.5 & 9.4 & 4.7 & 622.3 \\
\hline 5. & $\begin{array}{l}\text { Brachiaria deflexa (Schumach.) } \\
\text { C.E. Hubbard ex Robyns }\end{array}$ & 27 & 4 & 27.0 & 6.5 & 6.6 & 13.0 & 6.5 & 675.0 \\
\hline 6. & Cleome rutidosperma $\mathrm{DC}$. & 8 & 4 & 8.0 & 6.5 & 1.9 & 8.4 & 4.2 & 200.0 \\
\hline 7. & Commelina benghalensis L. & 24 & 4 & 24.0 & 6.5 & 5.8 & 12.3 & 6.1 & 600.0 \\
\hline 8. & Euphorbia heterophylla Linn. & 9 & 4 & 9.0 & 6.5 & 2.2 & 8.6 & 4.3 & 225.0 \\
\hline 9. & Euphorbia hirta Linn. & 6 & 3 & 8.0 & 4.8 & 1.9 & 6.8 & 3.4 & 266.7 \\
\hline 10. & $\begin{array}{l}\text { Mariscus alternifolius Vahl. (=M. } \\
\text { umbellatus Vahl.) }\end{array}$ & 50 & 5 & 40.0 & 8.1 & 9.7 & 17.8 & 8.9 & 800.0 \\
\hline 11. & Paspalum conjugatum Berg. & 37 & 4 & 37.0 & 6.5 & 9.0 & 15.5 & 7.7 & 925.0 \\
\hline 12. & $\begin{array}{l}\text { Peperomia pellucida (L.) H. B. } \\
\& \text { K. }\end{array}$ & 54 & 5 & 43.2 & 8.1 & 10.5 & 18.6 & 9.3 & 864.0 \\
\hline 13. & $\begin{array}{l}\text { Phyllanthus amarus Schum. \& } \\
\text { Thonn. }\end{array}$ & 5 & 3 & 6.7 & 4.8 & 1.6 & 6.5 & 3.2 & 222.3 \\
\hline 14. & Spigelia anthelmia Linn. & 7 & 5 & 5.6 & 8.1 & 1.4 & 9.4 & 4.7 & 112.0 \\
\hline 15. & Synedrella nodiflora Gaertn. & 178 & 5 & 142.4 & 8.1 & 34.7 & 42.7 & 21.4 & $2,848.0$ \\
\hline 16. & Talinum triangulare (Jacq.) Willd. & 6 & 4 & 6.0 & 6.5 & 1.5 & 7.9 & 4.0 & 150.0 \\
\hline
\end{tabular}

Table 6. Weed species distribution parameters in eggplant (NGB 01737)-oil palm intercropped plot at $6 \mathrm{WAI}$

\begin{tabular}{|c|c|c|c|c|c|c|c|c|c|}
\hline No. & Weed species & $\underset{\tilde{0}}{0}$ & 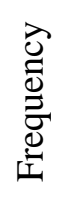 & 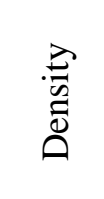 & 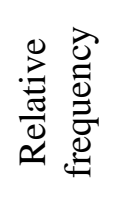 & 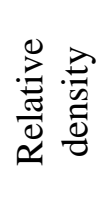 & 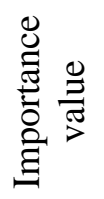 & 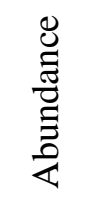 & 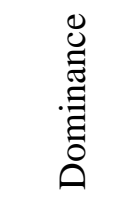 \\
\hline 1. & Ageratum conizoides Linn. & 113 & 5 & 90.4 & 11.1 & 27.3 & 38.4 & 19.2 & $1,808.0$ \\
\hline 2. & Borreria ocymoides (Burm. f.) DC. & 79 & 5 & 63.2 & 11.1 & 19.1 & 30.2 & 15.1 & $1,264.0$ \\
\hline 3. & Brachiaria lata (Schumach) C.E. & 1 & 1 & 4.0 & 2.2 & 1.2 & 3.4 & 1.7 & 400.0 \\
\hline 4. & Cleome rutidosperma DC. & 3 & 3 & 4.0 & 6.7 & 1.2 & 7.9 & 3.9 & 133.3 \\
\hline 5. & Commelina benghalensis L. & 29 & 5 & 23.2 & 11.1 & 7.0 & 18.1 & 9.1 & 464.0 \\
\hline 6. & $\begin{array}{l}\text { Mariscus alternifolius Vahl. (=M. } \\
\text { umbellatus Vahl.) }\end{array}$ & 4 & 4 & 4.0 & 8.9 & 1.2 & 10.1 & 5.0 & 100.0 \\
\hline
\end{tabular}


Table 6. Continued

\begin{tabular}{|c|c|c|c|c|c|c|c|c|}
\hline $\begin{array}{l}\text { 7. Peperomia pellucida (L.) H. B. } \\
\text { \& K. }\end{array}$ & 25 & 5 & 20.0 & 11.1 & 6.0 & 17.1 & 8.6 & 400.0 \\
\hline $\begin{array}{l}\text { 8. Phyllanthus amarus Schum. \& } \\
\text { Thonn. }\end{array}$ & 6 & 3 & 8.0 & 6.7 & 2.4 & 9.1 & 4.5 & 266.7 \\
\hline Setaria barbata (Lam.) Kunth & 110 & 5 & 88.0 & 11.1 & 26.5 & 37.7 & 18.8 & $1,760.0$ \\
\hline Spigelia anthelmia Linn. & 12 & 4 & 12.0 & 8.9 & 3.6 & 12.5 & 6.3 & 300.0 \\
\hline Talinum triangulare (Jacq.) Willd. & 5 & 3 & 6.7 & 6.7 & 2.0 & 8.7 & 4.3 & 222.2 \\
\hline Tridax procumbens Linn. & 4 & 2 & 8.0 & 4.4 & 2.4 & 6.9 & 3.4 & 400.0 \\
\hline
\end{tabular}

Table 7. Weed species distribution parameters in control plot at 3 WAI

\begin{tabular}{|c|c|c|c|c|c|c|c|c|}
\hline Weed species & $\stackrel{\pi}{0}$ & 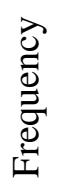 & 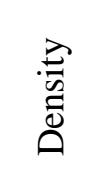 & 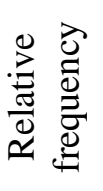 & 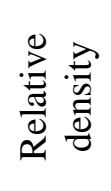 & 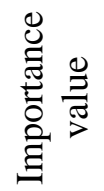 & 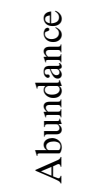 & 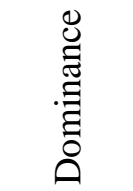 \\
\hline Ageratum conizoides Linn. & 269 & 5 & 53.8 & 7.7 & 16.1 & 23.8 & 11.9 & $1,076.0$ \\
\hline Bidens pilosa Linn. & 52 & 5 & 41.6 & 7.7 & 12.4 & 20.1 & 10.1 & 832.0 \\
\hline Borreria ocymoides (Burm. f.) DC. & 85 & 5 & 68.0 & 7.7 & 20.3 & 28.0 & 14.0 & $1,360.0$ \\
\hline $\begin{array}{l}\text { 4. Brachiaria deflexa (Schumach.) } \\
\text { C.E. Hubbard ex Robyns }\end{array}$ & 47 & 5 & 37.6 & 7.7 & 11.2 & 18.9 & 9.5 & 752.0 \\
\hline 5. Cleome rutidosperma DC. & 12 & 5 & 9.6 & 7.7 & 2.9 & 10.6 & 5.3 & 192.0 \\
\hline Commelina benghalensis L. & 4 & 3 & 5.3 & 4.6 & 1.6 & 6.2 & 3.1 & 177.7 \\
\hline 7. Digitaria horizontalis Willd. & 33 & 5 & 26.4 & 7.7 & 7.9 & 15.6 & 7.8 & 528.0 \\
\hline 8. Euphorbia hirta Linn. & 9 & 4 & 9.0 & 6.2 & 2.7 & 8.8 & 4.4 & 225.0 \\
\hline $\begin{array}{l}\text { 9. Mariscus alternifolius Vahl. (=M. } \\
\text { umbellatus Vahl.) }\end{array}$ & 29 & 5 & 23.2 & 7.7 & 6.9 & 14.6 & 7.3 & 464.0 \\
\hline $\begin{array}{l}\text { 10. Peperomia pellucida (L.) H. B. } \\
\& \text { K. }\end{array}$ & 34 & 5 & 27.2 & 7.7 & 8.1 & 15.8 & 7.9 & 544.0 \\
\hline 11. Spigelia anthelmia Linn. & 7 & 3 & 9.3 & 4.6 & 2.8 & 7.4 & 3.7 & 311.0 \\
\hline 12. Synedrella nodiflora Gaertn. & 14 & 4 & 14.0 & 6.2 & 4.2 & 10.3 & 5.2 & 350.0 \\
\hline 13. Talinum triangulare (Jacq.) Willd. & 7 & 3 & 9.3 & 4.6 & 2.8 & 7.4 & 3.7 & 311.0 \\
\hline
\end{tabular}

Table 8. Weed species distribution parameters in control plot at $6 \mathrm{WAI}$

\begin{tabular}{|c|c|c|c|c|c|c|c|c|c|}
\hline No. & Weed species & 吾 & 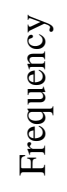 & 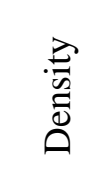 & 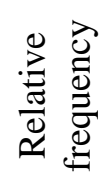 & 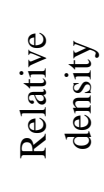 & 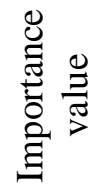 & 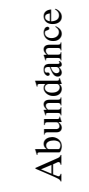 & 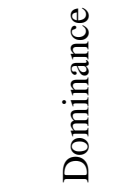 \\
\hline 1. & Ageratum conizoides Linn. & 107 & 5 & 85.6 & 9.4 & 15.5 & 25.0 & 12.5 & $\overline{1,712.0}$ \\
\hline 2. & Bidens pilosa Linn. & 154 & 5 & 123.2 & 9.4 & 22.4 & 31.8 & 15.9 & $2,464.0$ \\
\hline 3. & Borreria ocymoides (Burm. f.) DC. & 82 & 5 & 65.6 & 9.4 & 11.9 & 21.3 & 10.7 & $1,312.0$ \\
\hline 4. & Commelina benghalensis L. & 114 & 5 & 91.2 & 9.4 & 16.6 & 26.0 & 13.0 & $1,824.0$ \\
\hline 5. & Euphorbia hirta Linn. & 25 & 5 & 20.0 & 9.4 & 3.6 & 13.1 & 6.5 & 400.0 \\
\hline 6. & $\begin{array}{l}\text { Mariscus alternifolius Vahl. (=M. } \\
\text { umbellatus Vahl.) }\end{array}$ & 45 & 5 & 36.0 & 9.4 & 6.5 & 16.0 & 8.0 & 720.0 \\
\hline 7. & $\begin{array}{l}\text { Peperomia pellucida (L.) H. B. } \\
\& \text { K. }\end{array}$ & 13 & 4 & 13.0 & 7.5 & 2.4 & 9.9 & 5.0 & 325.0 \\
\hline 8. & Digitaria horizontalis Willd. & 2 & 2 & 4.0 & 3.8 & 0.7 & 4.5 & 2.3 & 200.0 \\
\hline & Setaria barbc & 109 & 5 & 87.2 & 9.4 & 15.8 & 25.3 & 12.6 & $1,744.0$ \\
\hline & Spigelia anthelmia Linn. & 9 & 4 & 9.0 & 7.5 & 1.6 & 9.2 & 4.6 & 225.0 \\
\hline
\end{tabular}


Table 8. Continued

\begin{tabular}{|c|c|c|c|c|c|c|c|c|}
\hline eq.) W & 7 & 4 & 7.0 & 7.5 & 1.3 & 8.8 & 4.4 & 175.0 \\
\hline ах ргоситье & 9 & 4 & 9.0 & 7.5 & 1.6 & 9.2 & 4.6 & 25.0 \\
\hline
\end{tabular}

Broadleaf weeds of families Asteraceae and Euphorbiaceae were noticed to have elevated numbers of members. This could be credited to the lifecycle, resources need and seed dispersal apparatus of these families of weeds. This agrees with a previous report by Olorunmaiye et al. (2011) who proposed high colonizing influence of these two families. This is further upheld by Oluwatobi and Olorunmaiye (2014), who recognized towering light necessity, aggressive growth, brief life cycle and enormous seed generation as qualities that may be responsible. Ageratum conizoides, Borreria ocymoides and Mariscus alternifolius were found to be predominant. This agrees with earlier study by Yakubu et al. (2006) and Karaye et al. (2007) who reported that several weeds and crops are site specific, while others will flourish over a broad array of habitat.

\section{Simpson's diversity indices of juvenile fruit vegetable-oil palm intercrops}

The tomato (NG/AA/SEP/09/053)-juvenile oil palm and the control plots recorded the peak and least weed species diversity (0.186 and 0.242$)$ respectively. The maximum diversity at $6 \mathrm{WAI}$ was obtained at the control plot with Simpson's Diversity Index of 0.123 (Table 9).

Table 9. Simpson's diversity indices of weed species in fruit vegetables-juvenile oil palm intercrop

\begin{tabular}{ccccccc}
\hline Simpson's & \multicolumn{3}{c}{ 3 WAI } & \multicolumn{3}{c}{ 6 WAI } \\
\cline { 2 - 7 } diversity & $\mathrm{D}$ & 1-D & 1/D & $\mathrm{D}$ & $1-\mathrm{D}$ & $1 / \mathrm{D}$ \\
\hline Plot A & 0.198 & 0.802 & 5.051 & 0.266 & 0.734 & 3.759 \\
Plot B & 0.186 & 0.814 & 5.376 & 0.173 & 0.827 & 5.780 \\
Plot C & 0.195 & 0.805 & 5.128 & 0.213 & 0.787 & 4.695 \\
Plot D & 0.242 & 0.758 & 4.132 & 0.123 & 0.877 & 8.130 \\
\hline
\end{tabular}

Note: Plots A = tomato (NGB 01665)-juvenile oil palm; B = tomato (NG/AA/SEP/09/053)-juvenile oil palm; $\mathrm{C}=$ eggplant (NGB 01737)-juvenile oil palm; $\mathrm{D}=$ control plots

Lower weed species diversity was noticed in control plots. This may be explained by the lack of canopy cover that would have provided shade as obtained in the intercropped plot. This is in harmony with a previous research by Baker et al. (2018), that intercropping practice facilitates effective weed control; with detrimental impacts on weed growth. Also, the study by Mhlanga et al. (2016) reported lessened weed diversity through prominent shading of weeds. Further, the research by Rahimi et al. (2019) is in unison with the finding of this work. They asserted higher diversity of weed species in intercropped plot than in monocrop.

\section{CONCLUSIONS}

This study revealed a progressive decline in the number of weed species composition at 3 through 6 WAI. Intercrop involving fruit vegetables and 2 -year-old oil palm culminated in greater abundance of broadleaf weed species than grass species. Broadleaf weed species most predominant belong to families Asteraceae and
Euphorbiaceae. Simultaneous cultivation of fruit vegetables within the fallow alley of immature oil palm should be promoted amid farmers, particularly at the earlier years prior to canopy closure.

\section{ACKNOWLEDGEMENT}

We would like to use this opportunity to recognize and thank Mr. Oke Tunde and Mr. Oke Temitope, for their support and provision during the conduction of this study.

\section{REFERENCES}

Abera, T. Feyisa, D. (2008). Faba bean and field pea seed proportion for intercropping system in Horro highlands of Western Ethiopia. African Crop Science Journal, 16 (4), 243-249. https://doi.org/10.4314/acsj.v16 i4.54398

Ademabayoje, O., Adigun, J., Adeyemi, O., Daramola, O., \& Ajiboye, G. (2020). Efficacy and economics of integrated weed 
management in groundnut (Arachis hypogea L.). Acta Agriculturae Slovenica, 116(1), 137144. https://doi.org/doi:10.14720/aas.2020.11 6.1 .1602

Akobundu, I. O., \& Agyakwa, C. W. (1998). A handbook of West African weeds. International Institute of Tropical Agriculture, Ibadan, Oyo State, Nigeria.

Ali, D., Ahmad, A., \& Mohsen, N. (2014). Determination of allelopathic effect of purple nutsedge (Cyperus rotundus) on germination and initial development of tomato (Lycopersicum esculentum). Indian Journal of Fundamental and Applied Life Sciences, 4(2), 576-580. Retrieved from http://www.cibtech. org/J-LIFE-SCIENCES/PUBLICATIONS/20 14/Vol-4-No-2/JLS-087-099-DADAR-DETE RMINATION-TOMATO.pdf

Alridiwirsah, A., Tampubolon, K., Sihombing, F. N., Siburian, E., Purba, Z., Wagino, Sulastri, Y. S., Manurung, I. R., Pratomo, B., Karim, S., Samosir, S. T. S., Supriyadi, Gustianty, L. R., \& Harahap, F. S. (2020). Glyphosate potassium salt dosage efficacy to weed control in Guava plants. Asian Journal of Plant Sciences, 19(4), 487-494. https://dx.doi.org/ 10.3923/ajps.2020.487.494

Amanullah, M. M., Somasundararam, E., Alagesan, A., Vaiyapuri, K., Pazhanivelan, S., \& Sathyamoorthi, K. (2006). Evaluation of some tree species for leaf fodder in Tamil Nadu. Research Journal of Agriculture and Biological Sciences, 2(6), 552-553. Retrieved from http://www.aensiweb.net/AENSIWEB/ rjabs/rjabs/2006/552-553.pdf

Audina, N. M., Maxiselly, Y., \& Rosniawaty, S. (2016). Pengaruh kerapatan naungan dan frekuensi penyiraman terhadap pertumbuhan bibit kemiri sunan (Reutealis trisperma (BLANCO) Airy Shaw). Jurnal Kultivasi, 15(2), 70-73. https://doi.org/10.24198/kulti vasi.v15i2.11901

Aynehband, A., Behrooz, M., \& Afshar, A. H. (2010). Study of intercropping agroecosystem productivity influenced by different crops and planting ratios. American-Eurasian Journal of Agricultural and Environmental Sciences, 7(2), 163-169. Retrieved from http://www. idosi.org/aejaes/jaes7(2)/7.pdf
Baker, C., Madakadze, I. C., Swanepoel, C. M., \& Mavunganidze, Z. (2018). Weed species composition and density under conservation agriculture with varying fertiliser rate. South African Journal of Plant and Soil, 35(5), 329 336. https://doi.org/10.1080/02571862.2018. 1431814

Devkota, A., \& Jha, P. K. (2010). Seed germination responses of the medicinal herb Centella asiatica. Brazilian Journal of Plant Physiology, 22(1), 143-150. https://doi.org/ 10.1590/S1677-04202010000200008

Dingaan, M. N. V., \& Du Preez, P. J. (2017). Floristic composition and species diversity of urban vegetation in Bloemfontein, Free State, South Africa. Bothalia, 47(1), 1-15. https://doi.org/10.4102/abc.v47i1.2244

Kanatas, P. J., Gazoulis, I., Travlos, I., Kakabouki, I., Kioussi, S., \& Babanioti, E. (2020). The effect of tillage on weed suppressive ability, leaf area, seed yield and protein content of Mucuna pruriens var. utilis. Notulae Botanicae Horti Agrobotanici ClujNapoca, 48(2), 871-881. https://doi.org/ $10.15835 /$ nbha48211887

Kanatas, P. J., Travlos, I. S., Gazoulis, J., Antonopoulos, N., Tsekoura, A., Tataridas, A., \& Zannopoulos, S. (2020). The combined effects of false seedbed technique, postemergence chemical control and cultivar on weed management and yield of barley in Greece. Phytoparasitica, 1(3), 83. https:// doi.org/10.1007/s12600-020-00783-x

Karaye, A. K., Yakubu, A. I., \& Aliyu, M. (2007). Checklist of weeds in irrigated garlic (Allium sativum) and onion (Allium cepa) in Sokoto River Valley. Journal of Research in Weed Science, 20, 53-57. Retrieved from https:// scholar.google.co.id/scholar?cluster $=1328725$ $6735502693456 \& \mathrm{hl}=\mathrm{id} \&$ as_sdt=2005\&sciodt $=0,5 \&$ authuser $=3$

Lawson, I. Y. D., Dzomeku, I. K., Asempa, R., \& Benson, S. (2006). Weed control in maize Mucuna and Canavalia as intercrops in the Northern Guinea Savanna Zone of Ghana. Journal of Agronomy, 5(4), 621-625. Retrieved from https://www.researchgate. net/publication/46026781_Weed_Control_in_ Maize_Using_Mucuna_and_Canavalia_as_Int ercrops_in_the_Northern_Guinea_Savanna_Z 
one_of_Ghana

Livesley, S. J., Ossola, A., Threlfall, C. G., Hahs, A. K., \& Williams, N. S. G. (2016). Soil carbon and carbon/nitrogen ratio change under tree canopy, tall grass, and turf grass areas of urban green space. Journal of Environmental Quality, 45(1), 215-223. https://doi.org/10.2134/jeq2015.03.0121

Mhlanga, B., Chauhan, B. S., \& Thierfelder, C. (2016). Weed management in maize using crop competition: a review. Crop Protection, 88, 28-36. https://doi.org/10.1016/j.cropro. 2016.05008

Ogunrayi, O. A., Akinseye, F. O., Goldberg, V., \& Bernhofer, C. (2016). Descriptive analysis of rainfall and temperature trends over Akure. Nigeria. Journal of Geography and Regional Planning, 9(11), 195-202. https://doi.org/ 10.5897/JGRP2016.0583

Okeyo, D. O., Fry, D. J., Bremer, D. J., Chandra, A., Genovesi, A. D., \& Engelke, M. C. (2014). Stolon growth and tillering of experimental zoysiagrasses in shade. HortScience, 49(11), 1444-1448. https://doi.org/10.21273/HORTS CI.46.10.1418

Olorunmaiye, P. M., Egbenrongbe, K. R., Adeoye, P. O., Alamu, O. O., \& Taiwo, S. (2011). Weed species composition of citrus-based cropping systems at National Horticultural Research Institute Ibadan, Nigeria. Agriculture and Biology Journal of North America, 2(529-537). https://doi.org/ 10.5251/abjna.2011.2.3.529.537

Olorunmaiye, P. M., Lagoke, S. T. O., Adigun, J. A., \& Orija, O. R. (2013). Effect of intercropping with maize on weed diversity in cassava. Environmental and Experimental Biology, 11, 189-193. Retrieved from http://eeb.lu.lv/EEB/201312/EEB_11_Olorun maiye.pdf

Oluwatobi, A. S., \& Olorunmaiye, K. S. (2014). Weed species distribution of juvenile oil palm tree (Elaeis guineensis) intercropped with maize (Zea mays), okra (Abelmoschus esculentus) and pepper (Capsicum anuumvar. abbreviatum). Notulae Scientia Biologicae, 6(4), 483-490. https://doi.org/10.15835/nsb 649358

Oluwatobi, A. S., Olorunmaiye, K. S., \& Adekola,
F. O. (2020). Growth assessment of juvenile oil palm intercropped with fruit vegetables in rainforest zone of Nigeria. Acta Agriculturae Slovenica, 116(2), 217-228. https://doi.org/ 10.14720/aas.2020.116.2.889

Pantilu, L. I., Mantiri, F. R., Ai, N. S., \& Pandiangan, D. (2012). Respons morfologi dan anatomi kecambah kacang kedelai (Glycine max (L.) Merill) terhadap intensitas cahaya yang berbeda. Journal of Bios Logos, 2(2), 81-87. https://doi.org/10.35799/jbl.2.2. 2012.1044

Peterson, K., Fry, J. D., \& Bremer, D. J. (2014). Growth responses of Zoysia spp. under tree shade in the Midwestern United States. HortScience, 49(11), 1444-1448. https://doi. org/10.21273/HORTSCI.49.11.1444

Rahayu, Syamsiyah, J., \& Nikmatus, L. (2020). Aggregate stability of alfisols root zone upon turfgrass treatment. Sains Tanah Journal of Soil Science and Agroclimatology, 17(1), 50-56. https://doi.org/10.20961/stjssa.v17i1. 40455

Rahimi, I., Amanullah, M. M., Ananthi, T., \& Mariappan, G. (2019). Influence of intercropping and weed management practices in weed parameters and yield of maize. International Journal of Current Microbiology and Applied Sciences, 8(4), 2167-2172. Retrieved from https://www.ijcmas.com/8-42019/Ishaq\%20Rahimi,\%20et\%20al.pdf

Rezig, F. A. M., Elhadi, E. A., \& Mubarak, A. R. (2012). Effect of incorporation of some wastes on wheat-guar rotation system on soil physical and chemical properties. International Journal of Recycling of Organic Waste in Agriculture, 1, 1-18. https:// doi.org/10.1186/2251-7715-1-1

Shadiul, H. B., Min, Y. E., Romij, M. D., Tea, S. P., Hang, W. K., Do, S. K., \& Park, K. W. (2011). Weed population dynamics under climatic change. Asian Journal of Turf Grass Science, 25(1), 174-182. http://dx.doi. org/10.5660/WTS.2014.3.3.174

Sherifi, E., \& Berisha, N. (2019). The effect of weed frequency in the overall alfalfa (Medicago sativa L.) productivity, case study from Kosovo. Acta Agriculturae Slovenica, 113(1), 41-49. http://dx.doi.org/10.14720/aas. 
2019.113.1.04

Suryaningsih, J. M., \& Darmadi, A. A. K. (2011). Inventarisasi gulma pada tanaman jagung (Zea mays L.) di lahan sawah Kelurahan Padang Galak, Denpasar Timur, Kodya Denpasar, Provinsi Bali. Jurnal Simbiosis, 1(1), 1-8. Retrieved from https://ojs.unud.ac.id/index. $\mathrm{php} /$ simbiosis/article/view/5494

Susanti, D., \& Safrina, D. (2018). Identifikasi luas daun spesifik dan indeks luas daun pegagan (Centella asiatica (L.) Urb.) di Karangpandan, Karanganyar, Jawa Tengah. Jurnal Tumbuhan Obat Indonesia, 11(1), 11-17. http://dx.doi. org/10.22435/toi.v11i1.8242. 11-17

Susanti, D., Safrina, D., \& Wijaya, N. R. (2021). Weed's vegetation analysis of centella
(Centella asiatica L. Urban) plantations. Caraka Tani: Journal of Sustainable Agriculture, 36(1), 110-122. http://dx.doi. org/10.20961/carakatani.v36i1.41269

Weber, J., Kunz, C., Peteinatos, G., Zikeli, S., \& Gerhards, R. (2017). Weed control using conventional tillage, reduced tillage, notillage, and cover crops in organic soybean. Agriculture, 7(5), 43. https://doi.org/10.3390/ agriculture 7050043

Yakubu, A. I., Alhassan, J., Lado, A., \& Sarkindiya, S. (2006). Comparative weed density studies in irrigated carrot (Daucus carota L.), potato (Solanum tuberosus) and wheat (Triticum aestivum Linn.) in Sokoto Rima Valley. Journal of Plant Science, 1(1), 14-21. https://doi.org/10.3923/jps.2006.14.21 